\title{
PRODUÇÃO DE LACASE FÚNGICA EM MEIO SÓLIDO UTILIZANDO RESÍDUOS FLORESTAIS COMO SUBSTRATO
}

\author{
N. V. M. MAXIMO ${ }^{1}$, A. C. FISCHBORN ${ }^{2}$, S. DUVOISIN Jr ${ }^{1}$ e P. M. ALBUQUERQUE ${ }^{1,2}$ \\ ${ }^{1}$ Universidade do Estado do Amazonas, Escola Superior de Tecnologia, Engenharia Química \\ ${ }^{2}$ Universidade do Estado do Amazonas, PPG em Biotecnologia e Recursos Naturais da Amazônia \\ E-mail para contato: nat14_maximo@hotmail.com
}

\begin{abstract}
RESUMO - A utilização de resíduos florestais para produção de enzimas oxidativas é uma alternativa para as indústrias obterem biocatalisadores com um custo mais baixo, como também uma maneira viável de agregar valor a estes resíduos, diminuindo seu impacto ambiental. Esse trabalho teve como objetivo estudar as condições de cultivo fúngico em estado sólido utilizando resíduos da hidrodestilação de plantas do gênero Aniba na obtenção lacase. Foram selecionadas as variáveis (umidade do substrato, fonte de nitrogênio, fonte de fósforo, tempo e temperatura de cultivo) que influenciam a atividade enzimática por meio de um planejamento experimental fracionado. $\mathrm{O}$ fungo UEA_105 cultivado no resíduo de preciosa e de pau-rosa obteve uma boa produção de lacase. Entretanto, o melhor resultado de atividade enzimática foi obtido com o fungo Aspergillus brasiliensis cultivado no resíduo de preciosa, utilizando níveis máximos de tempo, temperatura e fósforo e níveis mínimos de umidade e nitrogênio.
\end{abstract}

\section{INTRODUÇÃO}

As plantas da família Lauraceae apresentam-se amplamente distribuídas através das regiões tropicais e subtropicais do planeta, ocorrendo em todo o mundo, principalmente nas florestas da América Central e do Sul (Van Der Werff e Richter, 1996). Esta família compreende o gênero Aniba, o qual possui cerca de 40 espécies cuja maioria encontra-se na Amazônia. Algumas espécies são exploradas para a preparação de produtos medicinais, porém as espécies desse gênero são mais conhecidas pela produção e constituição dos seus óleos essenciais para a indústria de perfumaria, destacando-se a espécie Aniba roseadora Ducke, conhecida como pau-rosa, possuindo o linalol como principal constituinte do seu óleo essencial muito utilizado como fixador (Melo et al., 2006). Outras espécies, como A. canellila (H.B.K) Mez (preciosa) e A. parviflora (Meissn) Mez (macacaporanga) também são usadas na perfumaria (Marques, 2001).

Técnicas de extração dos compostos aromáticos das plantas, incluindo os óleos essenciais e os extratos, são citadas na literatura, entre elas a extração com solventes orgânicos, enfloração, prensagem ou utilização de $\mathrm{CO}_{2}$ supercrítico (Pybus e Sell, 1999; Simões et al., 2001). Pode ocorrer variação na composição do óleo obtido, em função do método de extração utilizado (Simões et al., 2001). 


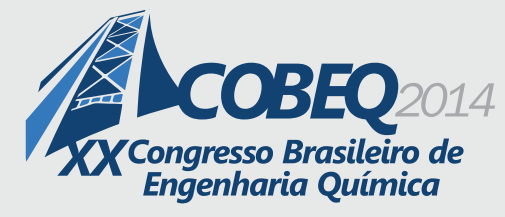

19 a 22 de outubro de 2014

Florianópolis/SC

A extração dos óleos essenciais gera resíduos sólidos que podem ser reaproveitados como substrato em processos biotecnológicos, na obtenção de compostos químicos e de produtos com valor comercial interessante como álcool, proteínas, ácidos orgânicos, entre outros (Pandey et al., 2000a). A prospecção química de metabólitos fúngicos apresenta-se como uma perspectiva interessante, pois estes microrganismos podem ser cultivados em larga escala e produzirem uma série de moléculas bioativas, como por exemplo, as enzimas (Takahashi e Lucas, 2008).

Muitas são as fontes de enzimas utilizadas industrialmente. Embora algumas sejam extraídas de tecidos animais e vegetais, as enzimas usadas na indústria são geralmente obtidas de microrganismos. A grande variedade e disponibilidade de bactérias, leveduras e fungos, aliada ao domínio das técnicas de cultivo, tornam as enzimas de origem microbiana as mais utilizadas em processos industriais. Apesar de um microrganismo ser capaz de produzir mais de mil enzimas distintas, é necessário um trabalho cuidadoso para o isolamento de espécies que produzam determinadas enzimas com as características desejadas (Faber, 2004).

A lacase (benzenediol: oxigênio oxidoredutase, EC 1.10.3.2) é uma enzima que catalisa a remoção de elétrons e prótons de grupos hidróxi-fenólicos e aminoaromáticos, formando radicais fenóxi livres e radicais amino, respectivamente (Leonowicz et al., 1999). Sua atividade tem sido demonstrada em mais de 60 tipos de fungos, em várias plantas superiores e em algumas bactérias (Mayer, 1987, Faure et al., 1994). Muitos pesquisadores procuram aperfeiçoar as condições de crescimento do fungo e a produção de lacase, tanto por métodos convencionais e/ou estatísticos, fazendo-se a combinação de diferentes fatores capazes de interferir na produção enzimática, para selecionar o método mais apropriado. Além disso, buscam melhor entender as propriedades da enzima a nível molecular e cinético, bem como a identificação mais eficiente de fontes produtoras de lacase (Giamreda et al., 1999; Ikehata et al., 2004).

A produção industrial de enzimas faz-se principalmente pelo processo de fermentação submersa (em meio líquido), que envolve o crescimento de microrganismos num meio rico e com elevadas concentrações de oxigênio. As enzimas também podem ser produzidas por fermentações em estado sólido (FES), as quais utilizam-se de substratos sólidos e são importantes economicamente para países como o Brasil, que possui abundância em biomassa e resíduos agroindustriais, que podem ser usados como matérias primas de baixo custo (Castilho et al., 2000). Esta técnica tem muitas vantagens sobre a fermentação líquida incluindo um rendimento superior, uma menor demanda de energia (Krishna, 2005), menor capital e custos de operação, além de utilizar equipamentos e meios de cultivo mais simples. Os resíduos podem ser reutilizados pela própria indústria que os produzem, principalmente como energia, ou podem ser vendidos para outras empresas e aplicados em usos diversos, como por exemplo, substrato para o cultivo de fungos. Se isto for feito, os resíduos deixam de ser um problema e passam a ser um subproduto da empresa em questão, podendo até gerar lucro (Lima e Silva, 2005).

Portanto, este trabalho teve como objetivo estudar as condições de cultivo fúngico em estado sólido utilizando resíduos da hidrodestilação de plantas do gênero Aniba como substrato para obter a máxima atividade de lacase, por meio de um planejamento experimental. 


\section{METODOLOGIA}

\subsection{Coleta do Material Vegetal e Obtenção do Resíduo Sólido}

Foram utilizados galhos e folhas das espécies Aniba parviflora (coletados na Fazenda Pematec em Santarém-PA), Aniba rosaeodora (coletados na Fazenda Magaldi em Maués-AM) e Aniba canellila (coletados na Reserva Ducke em Manaus-AM). Este material foi previamente seco e triturado, sendo utilizado para a extração do óleo essencial em hidrodestilador tipo Clevenger. O resíduo sólido obtido após a hidrodestilação foi na FES.

\subsection{Microrganismos}

Foi utilizado um fungo isolado da biodiversidade Amazônica, previamente selecionado como bom produtor de lacase (UEA_105) e que faz parte da micoteca do Programa de Pós-Graduação em Biotecnologia e Recursos Naturais da Amazônia (MBT-UEA). Também utilizou-se o fungo Aspergillus brasiliensis ATCC 16404. Os isolados foram mantidos a $5^{\circ} \mathrm{C}$ em meio batata-ágardextrose (BDA), e repicados periodicamente. Para o preparo do inóculo foi obtida uma suspensão de esporos $\left(1 \times 10^{8} \mathrm{UFC} / \mathrm{mL}\right)$.

\subsection{Cultivo dos Fungos Utilizando a Fermentação Sólida}

Para o cultivo fúngico foram utilizados frascos erlenmeyer de $125 \mathrm{~mL}$. O meio de cultivo sólido foi composto por $7 \mathrm{~g}$ de resíduo acrescidos de solução contendo os demais componentes do meio (fonte nitrogênio - $\mathrm{NH}_{4} \mathrm{NO}_{3}$ e fonte de fósforo $-\mathrm{KH}_{2} \mathrm{PO}_{4}$ ) com quantidade de água deionizada suficiente para atingir os valores de umidade estudados, sendo posteriormente autoclavado à $120^{\circ} \mathrm{C} / 15 \mathrm{~min}$. Em seguida, $50 \mu \mathrm{L}$ da suspensão de esporos foram inoculados no meio de cultivo sólido mantido a diferentes tempos e temperaturas em incubadora BOD, sem agitação.

Após o crescimento, $20 \mathrm{~mL}$ de água deionizada autoclavada foi adicionada ao meio, para facilitar a extração da enzima. Durante 2 min o meio contendo água foi colocado no ultrassom e por 10 min sob agitação em shaker. Foi realizada uma filtração a vácuo, em funil de Büchner e então as amostras foram congeladas para avaliação da atividade enzimática.

\subsection{Ensaio da Atividade Enzimática}

A medida da atividade enzimática foi realizada através de um método colorimétrico em espectrofotômetro UV-Vis. O aumento da absorbância em $530 \mathrm{~nm}$ em função da oxidação da seringaldazina foi relacionado ao aumento da atividade de lacase no extrato enzimático segundo a metodologia descrita por Ride (1980). Uma unidade de atividade enzimática (U) foi definida como a quantidade de enzima necessária para oxidar $1 \mathrm{mmol}$ de seringaldazina por minuto por $\mathrm{mL}$ de extrato enzimático. 


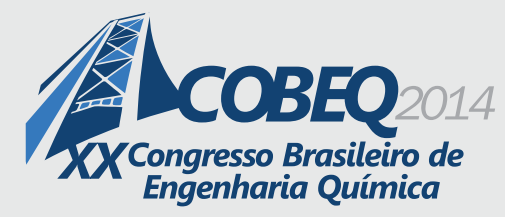

\subsection{Planejamento Experimental}

Um planejamento experimental fatorial fracionado $2^{5-1}$ foi realizado para selecionar as variáveis - umidade do substrato, adição de nutrientes (fontes de nitrogênio e de fósforo), tempo e temperatura de cultivo - que influenciam de forma estatisticamente significativa a atividade enzimática. A análise estatística dos dados experimentais foi realizada com a utilização do software Statistica 6.0. A matriz do planejamento experimental está apresentada na Tabela 1.

Tabela 1 - Matriz do planejamento experimental.

\begin{tabular}{c|c|c|c|c|c}
\cline { 2 - 6 } Ensaio & $\mathbf{T}\left({ }^{\circ} \mathbf{C}\right)$ & $\mathbf{t}$ (dias) & $\mathbf{N}(\%)$ & $\mathbf{P}(\%)$ & $\mathbf{U}(\%)$ \\
\hline $\mathbf{1}$ & $24(-1)$ & $9(-1)$ & $1,5(-1)$ & $3(-1)$ & $90(+1)$ \\
\hline $\mathbf{2}$ & $30(+1)$ & $9(-1)$ & $1,5(-1)$ & $3(-1)$ & $70(-1)$ \\
\hline $\mathbf{3}$ & $24(-1)$ & $15(+1)$ & $1,5(-1)$ & $3(-1)$ & $70(-1)$ \\
\hline $\mathbf{4}$ & $30(+1)$ & $15(+1)$ & $1,5(-1)$ & $3(-1)$ & $90(+1)$ \\
\hline $\mathbf{5}$ & $24(-1)$ & $9(-1)$ & $4,5(+1)$ & $3(-1)$ & $70(-1)$ \\
\hline $\mathbf{6}$ & $30(+1)$ & $9(-1)$ & $4,5(+1)$ & $3(-1)$ & $90(+1)$ \\
\hline $\mathbf{7}$ & $24(-1)$ & $15(+1)$ & $4,5(+1)$ & $3(-1)$ & $90(+1)$ \\
\hline $\mathbf{8}$ & $30(+1)$ & $15(+1)$ & $4,5(+1)$ & $3(-1)$ & $70(-1)$ \\
\hline $\mathbf{9}$ & $24(-1)$ & $9(-1)$ & $1,5(-1)$ & $6(+1)$ & $70(-1)$ \\
\hline $\mathbf{1 0}$ & $30(+1)$ & $9(-1)$ & $1,5(-1)$ & $6(+1)$ & $90(+1)$ \\
\hline $\mathbf{1 1}$ & $24(-1)$ & $15(+1)$ & $1,5(-1)$ & $6(+1)$ & $90(+1)$ \\
\hline $\mathbf{1 2}$ & $30(+1)$ & $15(+1)$ & $1,5(-1)$ & $6(+1)$ & $70(-1)$ \\
\hline $\mathbf{1 3}$ & $24(-1)$ & $9(-1)$ & $4,5(+1)$ & $6(+1)$ & $90(+1)$ \\
\hline $\mathbf{1 4}$ & $30(+1)$ & $9(-1)$ & $4,5(+1)$ & $6(+1)$ & $70(-1)$ \\
\hline $\mathbf{1 5}$ & $24(-1)$ & $15(+1)$ & $4,5(+1)$ & $6(+1)$ & $70(-1)$ \\
\hline $\mathbf{1 6}$ & $30(+1)$ & $15(+1)$ & $4,5(+1)$ & $6(+1)$ & $90(+1)$ \\
\hline $\mathbf{1 7}$ & $27(0)$ & $12(0)$ & $3(0)$ & $4,5(0)$ & $80(0)$ \\
\hline $\mathbf{1 8}$ & $27(0)$ & $12(0)$ & $3(0)$ & $4,5(0)$ & $80(0)$ \\
\hline $\mathbf{1 9}$ & $27(0)$ & $12(0)$ & $3(0)$ & $4,5(0)$ & $80(0)$ \\
\hline $\mathrm{T}=$ Temperatura; t $=$ tempo; N = fonte de Nitrogênio; P $=$ fonte de \\
fósforo; U = umidade. & & &
\end{tabular}

\section{RESULTADOS E DISCUSSÃO}

Os valores de atividade enzimática obtidos em cada experimento de FES utilizando os diferentes resíduos de Aniba e os dois fungos estão apresentados na Tabela 2.

Observa-se que para o resíduo de A. parviflora, obteve-se a maior atividade enzimática no ensaio 11 , com o fungo $A$. brasiliensis $(134 \mathrm{U} / \mathrm{mL})$, onde as condições de cultivo foram: $24^{\circ} \mathrm{C}, 15$ dias, $1,5 \%$ de nitrogênio, $6 \%$ de fósforo e $90 \%$ de umidade.

Para o resíduo de $A$. rosaeodora, obteve-se a maior atividade enzimática no ensaio 13 , com o fungo UEA_105 $(212 \mathrm{U} / \mathrm{mL})$, onde as condições de cultivo foram: $24^{\circ} \mathrm{C}, 9$ dias, $4,5 \%$ de nitrogênio, $6 \%$ de fósforo e $90 \%$ de umidade. 


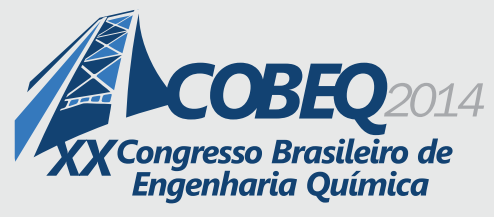

Para o resíduo de $A$. canelilla, obteve-se a maior atividade enzimática no ensaio 12 , com o fungo A. brasiliensis $(553 \mathrm{U} / \mathrm{mL})$, onde as condições de cultivo foram: $30^{\circ} \mathrm{C}, 15$ dias, 1,5\% de nitrogênio, $6 \%$ de fósforo e $70 \%$ de umidade.

Tabela 2 - Atividade enzimática $(\mathrm{U} / \mathrm{mL})$ de lacase obtida nos experimentos de FES com os fungos

UEA_105 e Aspergillus brasiliensis cultivados em resíduos de espécies do gênero Aniba.

\begin{tabular}{c|c|c|c|c|c|c}
\hline & \multicolumn{2}{|c|}{ A. parviflora } & \multicolumn{2}{c|}{ A. rosaedora } & \multicolumn{2}{c}{ A. canelilla } \\
\hline Ensaio & UEA_105 & A. brasiliensis & UEA_105 & A. brasiliensis & UEA_105 & A. brasiliensis \\
\hline 1 & $126 \pm 10$ & $124 \pm 6,0$ & $144 \pm 8,0$ & $118 \pm 26$ & $221 \pm 5,0$ & $146 \pm 2,0$ \\
\hline 2 & $104 \pm 2,0$ & $132 \pm 8,0$ & $168 \pm 8,0$ & $80 \pm 12$ & $166 \pm 12$ & $190 \pm 5,0$ \\
\hline 3 & $108 \pm 2,0$ & $112 \pm 4,0$ & $112 \pm 6,0$ & $64 \pm 30$ & $126 \pm 2,0$ & $189 \pm 3,0$ \\
\hline 4 & $100 \pm 0,0$ & $120 \pm 6,0$ & $133 \pm 5,0$ & $64 \pm 12$ & $127 \pm 1,0$ & $175,7 \pm 32$ \\
\hline 5 & $126 \pm 8,0$ & $110 \pm 4,0$ & $89 \pm 3,0$ & $89 \pm 9,0$ & $142 \pm 4,0$ & $147 \pm 5,0$ \\
\hline 6 & $128 \pm 0,0$ & $123 \pm 9,0$ & $87 \pm 7,0$ & $61 \pm 7,0$ & $151 \pm 7,0$ & $150 \pm 0,0$ \\
\hline 7 & $116 \pm 6,0$ & $113 \pm 1,0$ & $110 \pm 0,0$ & $63 \pm 5,0$ & $139 \pm 7,0$ & $177 \pm 3,0$ \\
\hline 8 & $108 \pm 8,0$ & $105 \pm 9,0$ & $108 \pm 4,0$ & $66 \pm 4,0$ & $125 \pm 1,0$ & $325 \pm 19$ \\
\hline 9 & $125 \pm 5,0$ & $114 \pm 10$ & $111 \pm 11$ & $70 \pm 8,0$ & $119 \pm 1,0$ & $155 \pm 1,0$ \\
\hline 10 & $123 \pm 5,0$ & $105 \pm 5,0$ & $84 \pm 6,0$ & $102 \pm 2,0$ & $115 \pm 1,0$ & $140 \pm 0,0$ \\
\hline 11 & $117 \pm 5,0$ & $134 \pm 2,0$ & $130 \pm 4,0$ & $70 \pm 0,0$ & $114 \pm 2,0$ & $204 \pm 18$ \\
\hline 12 & $109 \pm 7,0$ & $127 \pm 7,0$ & $109 \pm 5,0$ & $31 \pm 7,0$ & $127 \pm 5,0$ & $553 \pm 3,0$ \\
\hline 13 & $110 \pm 4,0$ & $100 \pm 10$ & $212 \pm 8,0$ & $89 \pm 5,0$ & $162 \pm 8,0$ & $162 \pm 14$ \\
\hline 14 & $128 \pm 2,0$ & $102 \pm 8,0$ & $104 \pm 8,0$ & $92 \pm 10$ & $145 \pm 7,0$ & $156 \pm 6,0$ \\
\hline 15 & $119 \pm 1,0$ & $93 \pm 9,0$ & $93 \pm 7,0$ & $79 \pm 11$ & $141 \pm 3,0$ & $203 \pm 7,0$ \\
\hline 16 & $124 \pm 10$ & $109 \pm 3,0$ & $104 \pm 2,0$ & $47 \pm 3,0$ & $139 \pm 5,0$ & $268 \pm 2,0$ \\
\hline 17 & $112 \pm 2,0$ & $98 \pm 8,0$ & $97 \pm 3,0$ & $60 \pm 12$ & $140 \pm 12$ & $142 \pm 10$ \\
\hline 18 & $109 \pm 1,0$ & $116 \pm 8,0$ & $111 \pm 3,0$ & $54 \pm 6,0$ & $126 \pm 6,0$ & $161 \pm 13$ \\
\hline 19 & $120 \pm 4,0$ & $117 \pm 5,0$ & $116 \pm 16$ & $41 \pm 9,0$ & $120 \pm 4,0$ & $150 \pm 12$ \\
\hline
\end{tabular}

Os diagramas de Pareto estão apresentados na Figuras 1 e 2.

Após a análise estatística, observa-se que para o fungo A. brasiliensis cultivado no resíduo de preciosa, o tempo de cultivo foi a variável estatisticamente mais significativa, e que maiores tempos de cultivo levam aos maiores valores de atividade enzimática. A temperatura também influenciou a produção da enzima de forma significativa, sendo que maiores temperaturas favorecem a produção de lacase. A umidade mostrou-se importante, sendo que os menores valores proporcionaram maiores atividades de lacase, da mesma forma que a adição de nitrogênio. Já o fósforo influenciou a produção de lacase de forma positiva, ou seja, maiores concentrações desse nutriente aumentaram a atividade enzimática. 


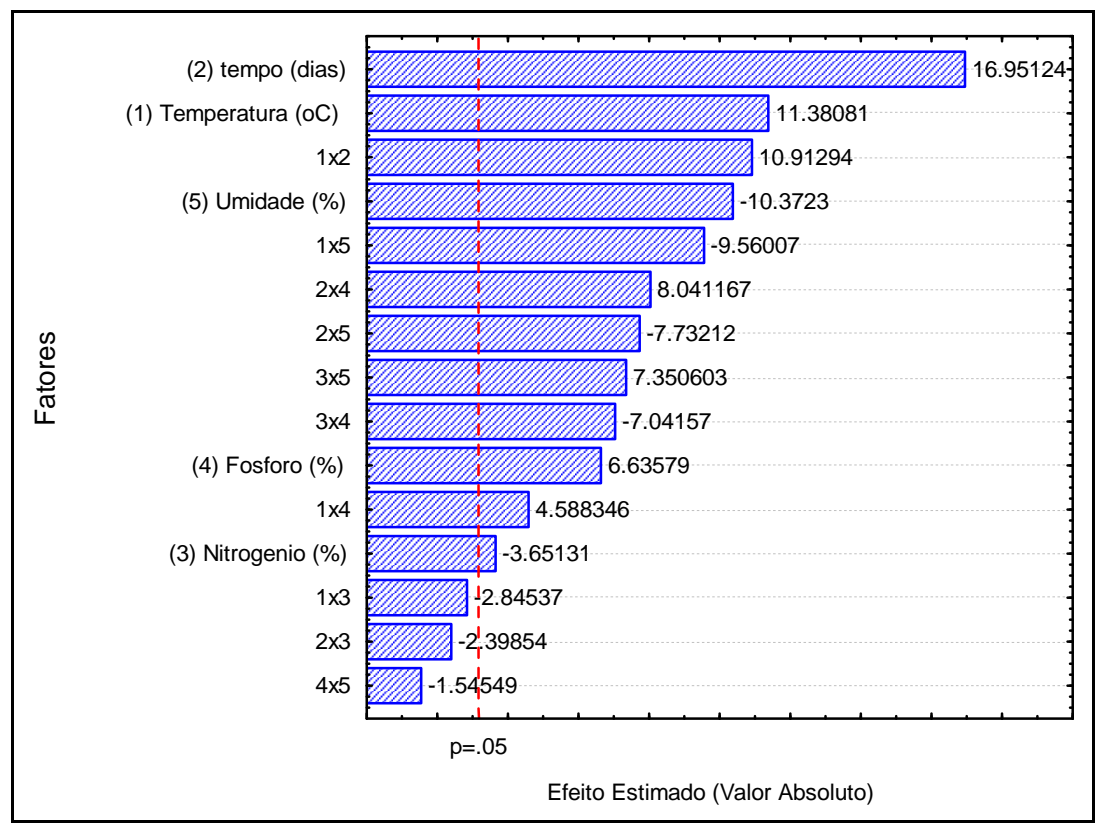

Figura 1 - Diagrama de Pareto obtido para o experimento de FES realizado com o resíduo de $A$. canelilla e o fungo Aspergillus brasiliensis.

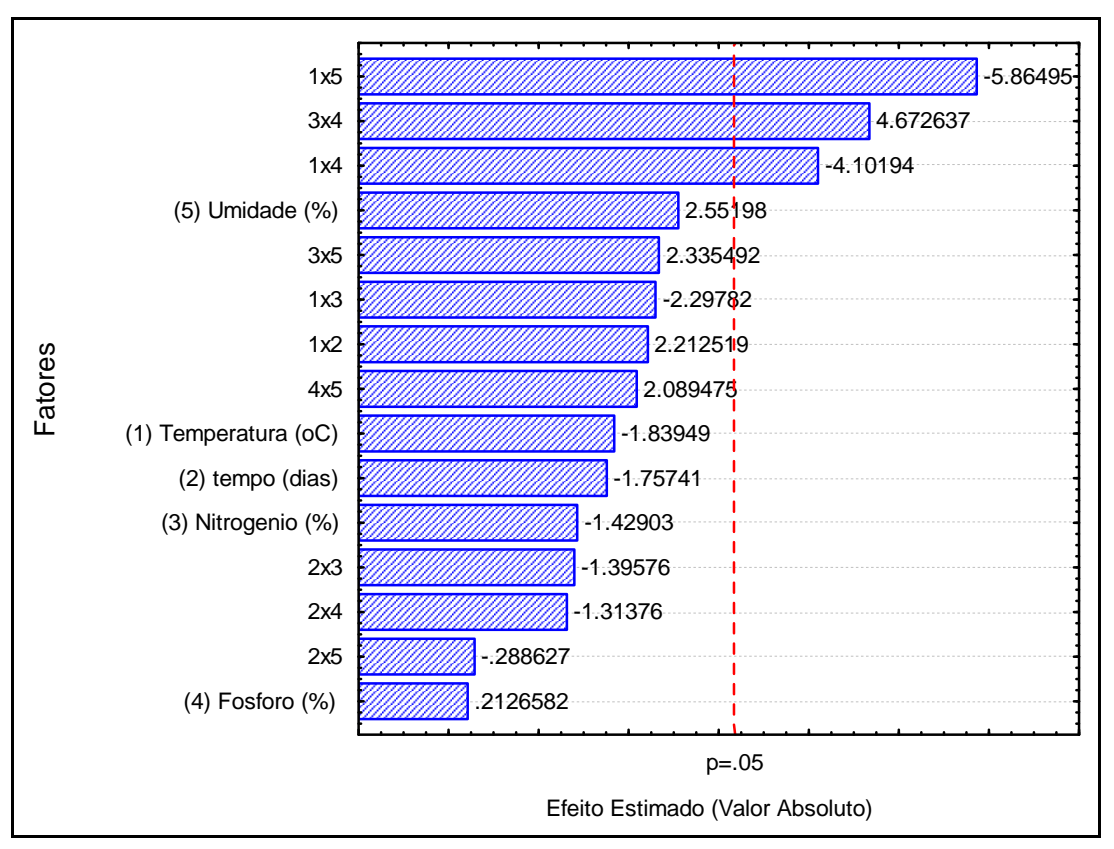

Figura 2 - Diagrama de Pareto obtido para o experimento de FES realizado com o resíduo de $A$. rosaeodora e o fungo UEA_105.

Para o fungo UEA_105 cultivado no resíduo de pau-rosa, observa-se que a umidade foi o fator 


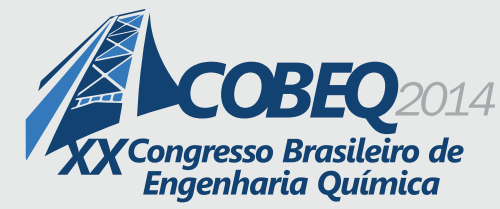

Engenharia Química
19 a 22 de outubro de 2014

Florianópolis/SC

mais importante, mas que não apresentou significância estatística. Apenas a interação entre os fatores influenciaram significativamente a produção de lacase.

Nos demais experimentos não foi observada significância estatística (95\% de confiança) para os fatores estudados sobre a atividade de lacase.

\section{CONCLUSÃO}

Foi possível aproveitar o resíduo sólido de espécies do gênero Aniba para a produção de lacase fúngica. A partir do planejamento experimental foi possível verificar a influência das variáveis Umidade, Tempo e Temperatura de Cultivo e concentrações de $\mathrm{N}$ e $\mathrm{P}$ sobre a atividade enzimática. Utilizando o resíduo de A. canelilla (preciosa) e o fungo A. brasiliensis foi possível obter o maior valor de atividade de lacase $(553 \mathrm{U} / \mathrm{mL})$.

\section{AGRADECIMENTOS}

Os autores agradecem à FAPEAM, CNPq e CAPES pelo suporte financeiro, ao INPA e à EST/UEA pela infraestrutura concedida para a realização deste trabalho.

\section{REFERENCIAS}

CASTILHO, L. R.; POLATO, C. M. S.; BARUQUE, E. A.; SANT'ANNA Jr., G. L.; CUNHA, M. A. A.; PAWLAK M. P.; FABBRIS, E. Z.; BARBOSA, A. M; DEKKER, R. F. H. Produção de exopolissacarídeo pelo fungo ascomiceto Botryosphaeria rhodina MM PI, VI Semana de Tecnologia em Alimentos, 2008.

FREIRE, D. Economic analysis of lipase production by Penicilium restrict um in sold-state and submerged fermentations. Biochem. Eng. J., v. 4, p. 239-247, 2000.

FABER, K. Biotransformations in organic chemistry. Springer: Berm. 2004.

FAURE, D.; BOUILLANT, M. L.; BALLY, R. Isolation of Azospirilum lipoferum 4t tn5 mutants affexted in melanization and laccase activity. Appl. Environ.l Microbiol., v. 60, p. 3413-3415, 1994.

GIANFREDA, S.; XU, F.; BOLLAG, J-M. Laccases a useful group of oxidoreductive enzymes. Bioremediation J., v. 3, n. 1, p. 1-125, 1999.

IKEHATA, K; BUCHANAN, I; SMITH, D. W. Recent developmens in the production of extracellular fungal peroxidades and laccases for waste treatment. J. Environ. Eng. Sci. v. 3, p. 1$19,2004$.

KRISHNA, C. Solid-state fermentation systems - an overview. Crit. Rev. Biotechnol, v. 25, p. 1$30,2005$.

LEONOWICZ, A.; MATUSZEWSKA, A.; LUTEREK, J.; ZIEGENHAGEM, D.; WOJTA WASILEWSKA, M.; CHO, N.-S.; HOFRICHTER, M.; ROGALSKI, J. Biodegradation of lignin by white-rot fungi. Fungal Gen. Biol., v. 27, p. 175-185, 1999. 


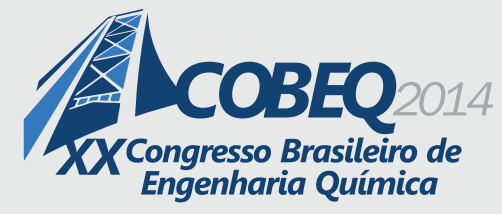

LIMA, E. G. de; SILVA, D. A. da. Resíduos gerados em indústrias de móveis de madeira situadas no pólo moveleiro de Arapongas-PR. Floresta, Curitiba, PR, v. 35, n. 1, 2005.

MACIEL, G. M. Desenvolvimento de bio processo para produção de xilanases por fermentação no estado sólido utilizando bagaço de cana de açúcar e farelo de soja. Dissertação de Mestrado. Programa de Pós Graduação em Processos Biotecnológicos, Universidade Federal do Paraná. Curitiba. 146p. 2006.

MARQUES, C. A. Importância Econômica da Familia Lauraceae Lindl. Floresta e Ambiente, Universidade Federal de Viçosa. v. 8, n. 1, p. 195-206, jan./dez. 2001.

MAYER, A. M. Polyphenol oxidases in plants-recent progress. Phytochem., v. 26 p. 11 - 20, 1987.

MELO, C. T.; MONTEIRO, A. P.; LEITE, C. P.; ARAWO, F. L.; LIMA, V. T.;BARBOSAFILHO, J. M.; FONTELES, F. M. M.; VASCONCELOS, S. M.; VIANA, B. G. S.; SOUSA, F. C. Anxiolyticlke effects of (O-methyl)-N-2,6- dihydroxybenzoyftyramine (apanese III) from Aniba riparia (Nees) Mez (Lauraceae) in mice. Biolog. Pharmac. Bul. v. 29, p. 451-454. 2006.

MENEZES, C. R.; SILVA, I. S.; INACIO, K. R.; PIUBELLI, F. A.; DURRANT, L. R. Utilização do bagaço de cana para a produção de enzimas lignocelulolíticas. Departamento de Ciência de Alimentos — Faculdade de Engenharia de Alimentos, UNICAMP, 2009.

PYBUS D. H.; SELL C. S. The Chemistry of Fragrances. Ed. The Royal Society of Chemistry: Cambridge, UK. 1999.

RIBEIRO, E. L. DA S.; HOPKINS, M. G.; VICENTINI, A.; SOTHERS, C. A. Flora da Reserva Ducke: Guia de identificação das plantas vasculares de uma floresta de terra-firme na Amazônia Central Manaus: INPA, 1999.

RIDE, J. P. The effects of induced lignification on the resistance of wheat cel walls to fungal degradation. Physiol. Plant Pathol., v. 16, p. 187-196, 1980.

SIMÕES, C. M. O.; SCHENKEL, E. P.; GOSMANN, G.; MELLO, J. C. P.; MENTZ, L. A.; PETROVICK, P. R. Farmacognosia: da planta ao medicamento. Porto Alegre/Florianópolis: URGS/ EDUFSC, 2001.

SUN, S. Y.; XU, Y.; WANG, D. Novel minor lipase from Rhizopus chinensis during solid-state fermentation: biochemical characterization and its esterification potential for ester synthesis. Bioresource Technol. v. 100, p. 2607-2612, 2009.

TAKAHASHI, J. A.; LUCAS, E. M. F. Ocorrência e diversidade estrutural de metabólitos fúngicos com atividade antibiótica. Quím. Nova, v. 31, n. 7. 2008.

TAVARES, A. M. P. Produção de lacase para potencial apicação como oxidante na indústria papaleira. Universidade de Aveiro, Departamento de química, 2006.

VAN DER WERFF, H.; RICHTER, H. G. Toward and improved classification of Lauraceae. Annals of the Missouri Botanical Garden, v. 8, p. 419-432. 1996. 